\title{
Retrospective, Multicentric Study of 180 Children with Cytochrome $c$ Oxidase Deficiency
}

\author{
MAREK BÖHM, EWA PRONICKA, ELŻBIETA KARCZMAREWICZ, MACIEJ PRONICKI, DOROTA PIEKUTOWSKA-ABRAMCZUK, \\ JOLANTA SYKUT-CEGIELSKA, HANNA MIERZEWSKA, HANA HANSIKOVA, KATERINA VESELA, MARKETA TESAROVA, \\ HANA HOUSTKOVA, JOSEF HOUSTEK, AND JIRI ZEMAN
}

\begin{abstract}
Department of Pediatrics [M.B., H.Ha., K.V., MT, H.Ho., J.Z.], Faculty of Medicine, Charles University, Prague, Czech Republic 12808; Departments of Paediatrics [E.P., J.S.-C., H.M.], Biochemistry and Experimental Medicine [E.K.], Pathology [M.P.], and Medical Genetics [D.P.A., E.P.], The Children's Memorial Health Institute, Warsaw, Poland 04730; Institute of Physiology [J.H.], Academy of Sciences of the Czech Republic, Prague, Czech Republic 14220
\end{abstract}

\begin{abstract}
A retrospective, multicenter study of 180 children with cytochrome $c$ oxidase (COX) deficiency analyzed the clinical features, prognosis, and molecular bases of the COX deficiency. Clinical symptoms including failure to thrive, encephalopathy, hypotony, Leigh syndrome, cardiac involvement, and hepatopathy appeared in most patients early after birth or in early childhood. Two thirds of all children died. Biochemical examination revealed an isolated COX deficiency in 101 children and COX deficiency combined with disturbances of other respiratory chain complexes in 79 children. Blood and cerebrospinal fluid lactate increased in $85 \%$ and $81 \%$ of examined cases, respectively. Pathogenic mutations in mitochondrial or nuclear DNA were established in 75 patients. Mutations in surfeit locus protein 1 gene (SURF1) were found in 47 children with Leigh syndrome; 2 bp deletion $845-846$ delCT was found in $89 \%$ of independent alleles. Mutations in a mitochondrial copper-binding protein $(\mathrm{SCO} 2)$ gene were found in nine children with encephalomyopathy and/or cardiomyopathy; all of them were homozygotes or heterozygotes for $1541 \mathrm{G}>\mathrm{A}$ mutation. Different mitochondrial DNA (mtDNA) deletion or depletion were found in nine children, mtDNA mutation $3243 \mathrm{~A}>\mathrm{G}$ in six, mtDNA mutation $8363 \mathrm{G}>\mathrm{A}$ in two children with Leigh syndrome and mtDNA mutations $8344 \mathrm{~A}>\mathrm{G}$, and 9205-9206delTA in one child each. COX deficiency represents a heterogeneous group of diseases with unfavorable prognosis. Marked prevalence of two nuclear DNA mutations (845-846delCT in the SURF1 gene and $1541 \mathrm{G}>\mathrm{A}$ in the SCO2 gene) associated with COX deficiency in a Slavonic population suggests the existence of regional differences in the genetic basis of COX deficiency. (Pediatr Res 59: 21-26, 2006)
\end{abstract}

$\mathrm{C}$ OX (respiratory chain complex IV, EC 1.9.3.1.) is the terminal enzyme of the mitochondrial respiratory chain that catalyses the transfer of reducing equivalents from cytochrome $c$ to molecular oxygen. This exergonic reaction is coupled with COX-mediated proton translocation from matrix to cytosol. Thus, the chemical energy of substrates is con-

Received March 2, 2005; Accepted July 5, 2005

Correspondence: Jiri Zeman, M.D., Ph.D., Department of Pediatrics, First Faculty of Medicine, Charles University, Ke Karlovu 2, 12808 Prague 2, Czech Republic; e-mail: jzem@lf1.cuni.cz

Supported by the European Community's Sixth Framework Programme for Research, Priority 1 "Life Sciences, Genomics and Biotechnology for Health" (LSHM-CT-2004503116), IGA NE/8065-3, GAC² 303/03/0749, MSM 0021620806, 1M6837805002, and PERFECT GLG1-CT-2002-90358.

DOI: 10.1203/01.pdr.0000190572.68191.13 verted into electrochemical potential of proton gradient $\Delta m \mathrm{H}^{+}$, which in turn drives adenosine triphosphate (ATP) synthesis by mitochondrial ATPase (1). The mammalian COX is a heterooligomer composed of 13 subunits (2). The three largest subunits forming the catalytic core of the enzyme are encoded by the mtDNA. The other 10 evolutionarily younger, nuclearly encoded subunits are involved in the assembly and regulation of the enzyme. The function of mammalian COX can be physiologically modulated by several ligands, and the enzyme represents important regulatory site of energy metabolism. The key event in this "second mechanism of respiratory control" is the phosphorylation of subunit IV and activity of phosphorylated COX is regulated at the level of efficiency of proton translocation by ATP/ADP ratio according to the ATP utilization (3).

Nuclear-encoded subunits IV, VIa, and VIIa of mammalian COX exist in tissue- and developmentally specific isoforms. Their role is related to specific energetic demands of different tissues and also to ontogenetic development. They differ in regulatory properties, which are well illustrated by subunit VIa. Heart isoform (VIaH) expressed in heart and skeletal muscle contains a binding site (sensor) for adenine nucleotides and at high ATP/ADP ratios the $\mathrm{H}^{+} / \mathrm{e}^{-}$stoichiometry of COX is decreased from 1 to 0.5 . The enzyme containing the liver isoform, which is found in most tissues, translocates protons at a low stoichiometry of 0.5 , independently of the ATP/ADP ratio (3). The dissipation of energy by COX containing VIaL is thought to be involved in thermogenesis (4). In addition, VIa subunit plays a role in the development of cardiac and skeletal muscle. At early embryonic stages, the VIaL isoform is predominant in all tissues, but increased VIaH expression is observed during differentiation of myogenic cells and late foetal stages of heart development (5).

Abbreviations: COX, cytochrome $c$ oxidase; CS, citrate synthase; mtDNA, mitochondrial deoxyribonucleic acid; SCO2, a mitochondrial copper-binding protein; SURF1, surfeit locus protein 1 
In addition to the structural subunits, numerous other proteins encoded by nuclear DNA are required for efficient assembly and maintenance of human COX (6). COX10 (farnesyl transferase) and COX15 are involved in the synthesis of heme A before its insertion into subunit I; SCO1, an $\mathrm{SCO}$, COX 11, and COX 17 control the incorporation of the copper atoms into subunits I and II, and SURF1 facilitates the assembly step from S2 to S3 intermediate, which is the addition of subunit II to a subunit I+IV subcomplex. Recently, an LRPPRC protein (leucine-rich pentatricopeptide repeat cassette) was identified that is involved in the translation or stability of the mRNA for mitochondrially encoded COX subunits (7).

Mammalian COX has been shown to exist in several native structural forms: monomers, dimers, and supercomplexes with respiratory chain complexes I and III. It is very likely that these forms also differ functionally. Increasing knowledge of the complexity of COX activity regulation in humans (which involves a large number of already known and probably many other yet unknown genes/proteins) helps to elucidate the cause of a large spectrum of clinical presentations of mitochondrial disorders associated with COX deficiency. In childhood, COX deficiency represents a heterogeneous group of disorders that predominantly affect tissues with a high-energy demand, especially the brain, muscle, and heart (8). Also genetically COX deficiency is rather complicated as the pathogenetic mutations are found in mtDNA as well as in nuclear genes (9).

Data from medical records of 180 COX-deficient children identified in Poland, Czech Republic, and Slovakia were analyzed in this retrospective, multicenter study to assess the clinical features, prognosis, and molecular bases of the COX deficiency in this region.

\section{METHODS}

Patients. This retrospective, multicenter study included all 180 COXdeficient children aged 1 mo to 18 y from 147 families identified within past 10 years in Poland, Czech Republic, and Slovakia, representing in total a population of 53 million inhabitants. Fifty-eight boys and 55 girls were born in Poland (113 cases/38 million inhabitants) and 36 boys and 31 girls were born in the Czech Republic and Slovakia (67 cases/15 million inhabitants). The overall male/female ratio was 1.09 . The medical reports from all 180 children at the time of diagnosis of COX deficiency were available in participating centers. After the diagnosis had been made, 138 children were followed at our institutions. No information about the present status of the remaining 42 children was available due to lost contact with the families and referring hospitals.

Ethics. The study was carried out in accordance with the Declaration of Helsinki of the World Medical Association and was approved by the Committees of Medical Ethics at all collaborating institutions. Informed consent was obtained from parents before any biopsies or molecular analyses were performed.

Clinical symptoms. The following clinical symptoms relevant to the course of the disease were defined: failure to thrive, a growth rate below 3 percentiles during infancy and early childhood and in older children markedly decreased growth rate crossing two major growth percentiles (i.e. from above the 75 th to below the 25th percentile) (10); encephalopathy, functional impairment of the central nervous system with developmental delay and/or regression, repeated seizures, pathologic pyramidal, extrapyramidal or cerebellar symptoms and cortical and periventricular atrophy, hypotonic syndrome with generalized peripheral hypo- or hyperreflexia, Leigh syndrome with typical symmetric necrotic lesions in basal ganglia and/or in the brainstem using magnetic resonance imaging or at autopsy; cardiac involvement, a "generally or partially hypertrophic" left ventricle in two-dimensional echoDoppler investigation, changed dimension of the left ventricle posterior wall, and/or the intraventricular septum in diastole measured by $\mathrm{M}$ mode beyond 2 SD (hypertrophic cardiomyopathy) (11), or isolated heart conduction abnor- malities; hepatopathy, acute and chronic liver disease (liver failure, fibrosis, cirrhosis, steatosis) or isolated persistent increase in serum alanineaminotransferase and aspartate-aminotransferase without elevation of serum creatine kinase; endocrinopathy, such as diabetes mellitus, hypothyroidism, pituitary dysfunction, or adrenal insufficiency; and nephropathy, presenting as tubulopathy (proximal disfunction of Fanconi type, hypomagnesuria) or progressive renal failure.

Biochemical analyses. In all patients, COX deficiency was analyzed biochemically in skeletal muscle and/or cultivated fibroblasts. In some patients, the deficiency was also detected in other tissues. COX deficiency was defined as a decreased activity $<30 \%$ with decreased COX/CS (citrate synthase) ratio $<30 \%$ of the mean of age-related controls (12). The diagnostic procedures have been improved in the study period. First, enzymatic assay in muscle homogenates was applied and/or histochemical COX staining was performed. Later on, cultivated fibroblasts and isolated muscle mitochondria were used for enzymatic analyses. Muscle mitochondria were isolated according to Makinen and Lee (13) without the use of protease. Respiratory chain complex, NADH-coenzyme $\mathrm{Q}_{10}$ oxidoreductase (complex I), succinatecoenzyme $\mathrm{Q}_{10}$ oxidoreductase (complex II), coenzyme $\mathrm{Q}_{10}$-cytochrome $c$ oxidoreductase (complex III), COX (complex IV) and NADH-cytochrome $c$ reductase (complex I+III), and CS activity was measured with spectrophotometry $(14,15)$. Protein was determined by the method of Lowry et al. (16). The ratio between the activity of individual respiratory chain complexes and CS was calculated to eliminate the possible effect of changes in the number of mitochondria in patient cells. The quality of the enzymatic assays protocol was confirmed by the 1st European laboratory ring external quality test (Dr. Gellerich, Martin Luther University, Halle, Germany). Two-dimensional electrophoresis (BN-PAGE/SDS-PAGE) and/or Western blot analysis $(17,18)$ was performed to assess the amount and composition of respiratory chain complexes. In cases of Polish patients, respiratory chain complexes activity was measured in tissue homogenates as above and the procedure was not changed during the study. Only the COX deficiency analyses by two independent methods (spectrophotometry and histochemistry) in fibroblasts and muscle were included in the study.

Molecular methods. One or more molecular analyses were performed in 170 children. In most patients, the PCR screening for large deletions in mtDNA and PCR-RFLP analyses for mtDNA mutations 3243A $>G$ and $8344 \mathrm{~A}>\mathrm{G}$ were performed. Patients with negative screening were divided into smaller groups according to their clinical profiles. With the use of cyclic sequencing, SURF1, SCO2, SCO1, and COX1O genes or whole mtDNA were analyzed in corresponding groups. Southern blot analyses were used for identification of large-scale deletions in mtDNA or mtDNA depletion. Not all currently indicated and available DNA analyses were performed in each of the COX-deficient children due to inaccessibility or insufficient amounts of DNA samples.

\section{RESULTS}

The age at the onset, the frequency of clinical symptoms, and the course of the disease in 180 children with COX deficiency are shown in Tables 1 and 2. An underlying genetic defect was detected in 56 of 101 patients with isolated COX deficiency and 19 of 79 patients with COX deficiency combined with disturbances of other respiratory chain complexes. The results of molecular analyses in probands are shown in Table 3. Many of the patients had normal birth weight and length and deteriorated after respiratory tract infection or other stress-bearing events. Generally, the first symptoms were failure to thrive and hypotony.

Progressive encephalopathy was observed in most of the patients, but the kind of neurologic symptoms and their severity differed largely. As apparent from Table 1, Leigh syndrome was found in all SURF1 patients, none of SCO2 patients, and only in four of 43 cases of isolated COX defect without known DNA mutation. In combined defects, only four cases of 79 patients presented with Leigh syndrome, two of which harbored mtDNA mutation $8363 \mathrm{G}>\mathrm{A}$. In other patients, the neurologic features and neuroimaging were not specific for mitochondrial disorder, and a muscle biopsy was 
Table 1. Frequency of clinical symptoms in 180 children with isolated or combined COX deficiency

\begin{tabular}{|c|c|c|c|c|c|c|}
\hline $\begin{array}{l}\text { COX deficiency } \\
\text { mutations }\end{array}$ & \multicolumn{3}{|c|}{ Isolated $(n=101)$} & \multicolumn{2}{|c|}{ Combined* $(n=79)$} & Total \\
\hline Affected families & 35 & 6 & 35 & 19 & 52 & 147 \\
\hline Failure to thrive & $40 / 46(87 \%)$ & $6 / 8(75 \%)$ & $22 / 38(58 \%)$ & $16 / 19(84 \%)$ & $22 / 48(46 \%)$ & $106 / 159(67 \%)$ \\
\hline Hypotony & $41 / 46(89 \%)$ & $9 / 9(100 \%)$ & $27 / 39(69 \%)$ & $10 / 18(56 \%)$ & $30 / 48(63 \%)$ & $117 / 160(73 \%)$ \\
\hline Cardiac involvement & $4 / 47(9 \%)$ & $4 / 9(44 \%)$ & $8 / 37(22 \%)$ & $11 / 19(58 \%)$ & $12 / 53(23 \%)$ & $39 / 165(24 \%)$ \\
\hline Hepatopathy & $0 / 46$ & $3 / 9(33 \%)$ & $17 / 39(44 \%)$ & $4 / 19(21 \%)$ & $16 / 54(30 \%)$ & $40 / 167(24 \%)$ \\
\hline Endocrinopathy & $0 / 47$ & $0 / 8$ & $1 / 39(3 \%)$ & $6 / 19(32 \%)$ & $2 / 54(4 \%)$ & $9 / 167(5 \%)$ \\
\hline Fanconi syndrome & $0 / 47$ & $0 / 9$ & $1 / 38(3 \%)$ & $0 / 19$ & $1 / 37(3 \%)$ & $2 / 150(1 \%)$ \\
\hline Renal failure & $0 / 47$ & $0 / 9$ & $1 / 38(3 \%)$ & $2 / 19(11 \%)$ & $4 / 55(7 \%)$ & $7 / 168(4 \%)$ \\
\hline
\end{tabular}

* COX deficiency combined with disturbances of one or more other respiratory chain complexes.

$\dagger$ Patients with encephalopathy and bilateral necrotic lesions in basal ganglia and/or in the brainstem recognized by magnetic resonance imaging or at autopsy.

Table 2. Age at onset of clinical symptoms and the course of the disease in 180 children with isolated or combined COX deficiency

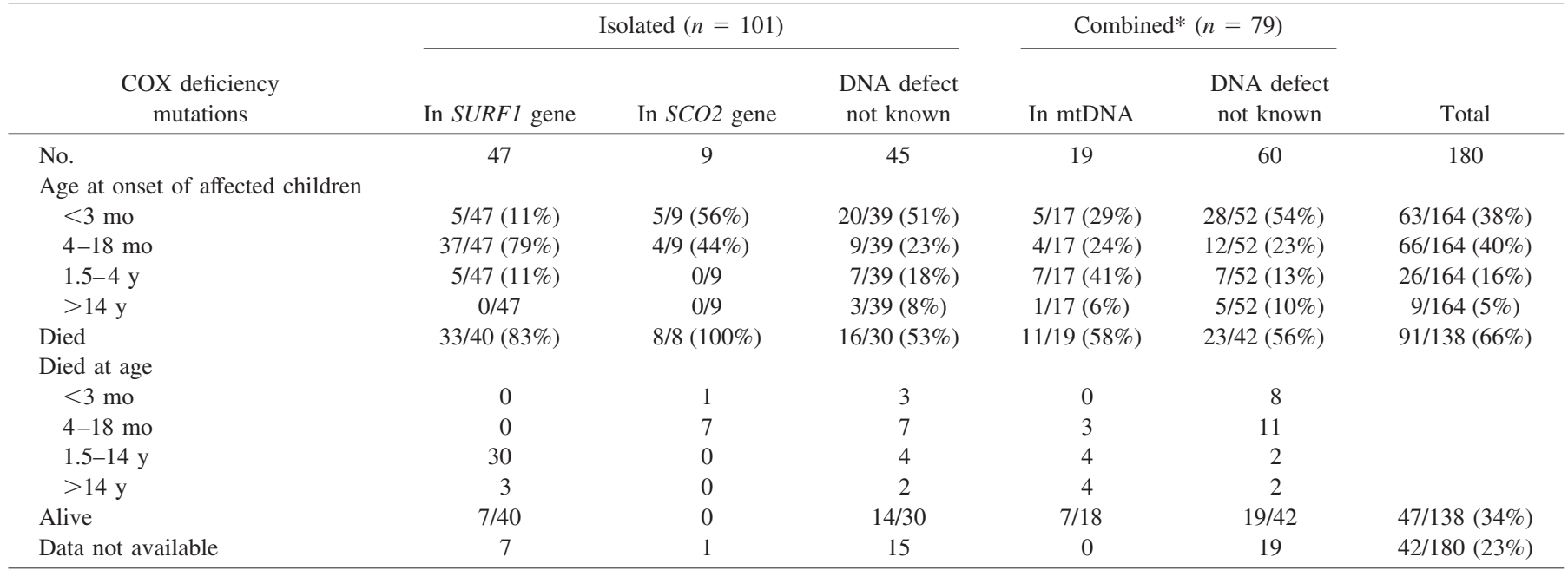

* COX deficiency combined with disturbances of one or more other respiratory chain complexes.

performed due to the coexistence of increased blood lactate. MELAS-like stroke episodes and progressive external ophthalmoplegia were seen only in a few adolescents. Hypertrichosis was a common finding, especially in children with Leigh syndrome. The course of the disease was usually progressive and unfavorable; $66 \%$ of patients died.

Increased blood lactate was found in $85 \%$ and increased blood alanine in $65 \%$ of patients. The CSF lactate level was elevated in $81 \%$ of examined cases. Lactic acidosis occurred regularly in patients with combined respiratory chain defects and known mtDNA mutations. In the children with Leigh syndrome, increased lactate level was associated often with respiratory alkalosis and hypocapnia (19). There were some SURF1-deficient Leigh patients without lactic acidosis (10\%), with normal lactate concentration in cerebrospinal fluid (8\%).

Mutations in the SURF1 gene occurred exclusively in children with Leigh syndrome; the 2bp deletion 845-846delCT in SURF1 gene was detected in $89 \%$ of independent alleles (Table 3). The mutations in the $\mathrm{SCO} 2$ gene were identified in nine children with encephalomyopathy and/or cardiomyopathy, the mutation $1541 \mathrm{G}>\mathrm{A}$ in $\mathrm{SCO} 2$ was found in $83 \%$ of independent alleles. mtDNA mutations were found in 19 children with combined COX deficiency. The heteroplasmic mtDNA mutation 3243A $>\mathrm{G}$ was present in six unrelated children, mtDNA mutation $8363 \mathrm{G}>\mathrm{A}$ was found in two unrelated children with Leigh syndrome, and mtDNA mutation 8344A $>$ G (20) and 9205-9206delTA (21) in one child each. The same mtDNA mutations with various levels of heteroplasmy were detected in the patients' mothers and other maternal relatives (not included in the study). mtDNA largescale deletions were found in eight children with multiorgan involvement including encephalomyopathy, endocrinopathy, and heart conduction impairment and in one child with Pearson syndrome (Table 3). All of them were sporadic and heteroplasmic deletions; in five cases, we found single deletions of 4.9-5.6 kb, in one child deletion/insertion was found and two cases harbored multiple mtDNA deletions. mtDNA depletion was detected in a boy with Alpers syndrome (22).

The missing molecular diagnosis in our group of patients was due to technical problems due to accessibility of material or the underlying genetic defect is still not known. Among children with COX deficiency without known molecular defect, we found both mild and severe reduction of COX activity as well as differences in severity of clinical phenotype. 
Table 3. Frequency of mutations in SURF1 and SCO2 genes in 41 probands with isolated COX deficiency and the frequency of mtDNA mutations in 19 probands with combined COX deficiency (data in siblings are not shown)

\begin{tabular}{|c|c|c|c|c|}
\hline & Gene & Probands & Mutation type & Frequency \\
\hline \multirow[t]{11}{*}{ nDNA } & SURF1* & 35 & 845-846delCT/845-846delCT & $18 \mathrm{x}$ \\
\hline & & & 845-846delCT/? & $6 x$ \\
\hline & & & 845-846delCT/312-321del10insAT & $3 \mathrm{x}$ \\
\hline & & & 845-846delCT/756delCA & $1 \mathrm{x}$ \\
\hline & & & 845-846delCT/704T $>\mathrm{C}$ & $1 \mathrm{x}$ \\
\hline & & & $845-846 \mathrm{delCT} / 821 \mathrm{~A}>\mathrm{G}$ & $1 \mathrm{x}$ \\
\hline & & & 312-321del10insAT/? & $1 \mathrm{x}$ \\
\hline & & & $688 \mathrm{C}>\mathrm{T} / 688 \mathrm{C}>\mathrm{T}$ & $1 \mathrm{x}$ \\
\hline & $\mathrm{SCO} 2 \dagger$ & 6 & $1541 \mathrm{G}>\mathrm{A} / 1541 \mathrm{G}>\mathrm{A}$ & $4 \mathrm{x}$ \\
\hline & & & $1541 \mathrm{G}>\mathrm{A} / 1280 \mathrm{C}>\mathrm{T}$ & $1 \mathrm{x}$ \\
\hline & & & $1541 \mathrm{G}>\mathrm{A} / 1518 \mathrm{del} \mathrm{A}$ & $1 \mathrm{x}$ \\
\hline \multirow[t]{5}{*}{ mtDNA } & Point mutation $\$$ & 10 & $3243 \mathrm{~A}>\mathrm{G}$ MELAS & $6 x$ \\
\hline & & & Deletion/Insertion 8035 bp, heteroplasy $80 \%$ & $1 \mathrm{x}$ \\
\hline & & & Multiple deletion (long-range PCR) heteroplasmy $<10 \%$ & $2 \mathrm{x}$ \\
\hline & & & $\begin{array}{l}\text { PCR screening of the common deletion } 5,6 \mathrm{~kb} \text { in the region } \\
\text { of mtDNA } 8150-14276 \mathrm{bp}\end{array}$ & $1 \mathrm{x}$ \\
\hline & mtDNA depletion & 1 & $\begin{array}{l}\text { In liver and brain, the mtDNA amount was reduced to } 11 \\
\text { and } 15 \%\end{array}$ & $1 \mathrm{x}$ \\
\hline
\end{tabular}

* Mutation in SURF1 gene was found in 35 probands and 12 affected siblings.

$\dagger$ Mutation in SCO2 gene was found in six probands and three affected siblings.

$\ddagger$ mtDNA mutations were also found in mothers and other maternal relatives.

$\S \mathrm{mtDNA}$ deletion/duplication was not found in parents.

\section{DISCUSSION}

Clinical manifestation. The age at onset of clinical symptoms in patients with disturbances of oxidative phosphorylation system (OXPHOS) is extremely variable, but OXPHOS disorders in childhood are characterized by early onset of the disease in general $(8,23)$. The true prevalence of mitochondrial disorders in Europe is uncertain, but the minimum prevalence of clinically affected adults with mtDNA point mutations or deletions in the northeast of England is 1:10,000. The prevalence of those affected or at risk is even 2.9:10 000 (24). Our study in 180 children with COX deficiency goes back $10 \mathrm{y}$ and also includes patients referred to us by other hospitals. Therefore, not all children underwent full clinical and biochemical evaluation. Nevertheless, this study represents one of the largest groups of children with COX deficiency described so far. About 16 new COX-deficient children are identified yearly in Poland and the Czech and Slovak Republics. This may suggest the frequency of 1:35,000 births, but we are aware that this figure is underestimated.

Similarly to other studies $(25,26)$, the most common clinical problems in our group of children with COX deficiency are the functional impairments of the brain, muscle, and heart. From a clinical point of view, the major and most important discriminator for mitochondrial disorders with COX deficiency in childhood is encephalopathy characterized as functional impairment of the central nervous system combined with failure to thrive and any impairment of any other tissue or even more tissues. Quite often the encephalopathy presents specifically with typical bilateral necrotic lesions in the basal ganglia or brainstem characterized as Leigh syndrome, which may discriminate subgroups with the most severe COX defects. Interestingly, Leigh syndrome was almost exclusively found in patients with mutations found in SURF1 gene.

In our children, the first clinical symptoms developed early after the birth in more than one third of patients and $78 \%$ of patients were already clinically affected within the first 18 mo of life. Children with earlier onset of the disease, especially patients with Leigh syndrome due to mutations in the SURF1 gene and patients with encephalopathy and cardiomyopathy due to mutations in the $\mathrm{SCO} 2$ gene, developed more severe course of the disease, as was previously described (23). They also died earlier than the children with combined respiratory chain defects.

Hypertrophic cardiomyopathy was found in $24 \%$ of our patients, similar to observations of other authors $(9,11)$. In contrast to other reports (23), echocardiographic investigations revealed mild hypertrophic cardiomyopathy also in four of 47 children with Leigh syndrome due to mutations in the SURF1 gene, but cardiomyopathy developed only in children surviving more than $10 \mathrm{y}$, whereas the majority of children with Leigh syndrome died much earlier. One boy with lateonset cardiomyopathy of unknown molecular background un- 
derwent heart transplantation before COX deficiency was established in his affected younger brother. Conduction heart defects, although common in adult patients with mitochondrial disorders, were found in our cohort in only eight children older than $7 \mathrm{y}$ with a large-scale mtDNA deletion or common MELAS mutation. However, recently, mitochondrial cardiomyopathy has been more often suspected by local physicians referring children to us with multiorgan involvement and hyperlactatemia for diagnosis. In our patients, eyelid ptosis and progressive external ophthalmoplegia were rare.

Renal involvement in children with COX deficiency is quite rare. We observed chronic renal insufficiency in less than 5\% of patients. One boy with Kearns-Sayre syndrome underwent kidney transplantation before the diagnosis of COX deficiency was made and mtDNA deletion was recognized. Fanconi syndrome is also quite rare in children with mitochondrial disorders. Caruso et al. (27) described Fanconi syndrome in three of 60 patients with respiratory chain defects; we have seen it only in two of 180 children. However, the results of our retrospective study indicated that most of COX-deficient patients in our study were not investigated properly to determine mild dysfunction of renal tubules.

The prognosis in children with COX deficiency is generally unfavorable, the course of the disease is usually progressive, and the current therapeutic possibilities are very limited. Vitamin cocktails, coenzyme $\mathrm{Q}_{10}$, and sodium-dichloroacetate were tried in our patients without any significant clinical improvement, and $66 \%$ of our patients died in childhood, nearly half of them within the first 18 mo of life. A similarly poor prognosis was observed in Italian patients with COX deficiency; $50 \%$ of them died in early childhood (27).

Biochemical analyses. In children with mitochondrial disorders, the clinical course of the disease may be associated with excessive production of lactic acid and development of metabolic acidosis. Hyperlactacidemia was found in more than $80 \%$ of our children, usually together with an increased lactate:pyruvate ratio. Biochemical diagnoses in children with the clinical suspicion on COX deficiency usually rely on spectrophotometric or polarographic analyses of COX activity, especially in muscle biopsies or cultivated fibroblasts. The analyses in isolated muscle mitochondria from the fresh muscle biopsy as done in one of our laboratories are preferred, but analyses of muscle homogenate from frozen muscle biopsy have also a diagnostic value in routine practice. In accordance with other studies, the most profound decrease of COX activity both in muscle tissue and fibroblasts was found in all children with mutations in SURF1 gene. In all patients with mutations in $\mathrm{SCO} 2$ gene, COX activity was low in muscle tissue but practically normal in the cultivated fibroblasts. In other cases, the spectrum of enzymatic changes was very broad and included COX defects that were more pronounced in muscle as well as defects that dominated in fibroblasts.

DNA analyses. A significant percentage of consanguinity in affected families of COX-deficient children emphasized in some reports (25) indicates the major role of nDNA mutations in comparison with mtDNA mutation in this age group, but in our study, consanguinity was not a common finding. Genetic counseling in families with COX deficiency may be difficult, especially if the diagnosis in the proband was confirmed only at the enzymatic level. A molecular defect of COX deficiency is usually recognized in fewer than half of the patients. mtDNA mutations in children with isolated COX deficiency were described in mtDNA genes encoding the three main COX subunits I, II, and III, but these mtDNA mutations are quite rare (28). Until now, no mutations in nuclear genes encoding 10 other structural COX subunits were described.

In contrast, an increasing number of mutations in genes encoding the COX assembly-specific factors are recognized as a cause of isolated COX deficiency in childhood. They include mutations in the SURF1 gene in children with Leigh syndrome (29), mutations in the SCOl gene in neonates with acute liver failure (30), mutations in the $\mathrm{SCO} 2$ gene in children with fatal hypertrophic cardiomyopathy and encephalopathy (31), mutations in the COX10 gene in children with tubulopathy (30), mutations in the COX15 gene in the child with cardiomyopathy (32), and mutations in the LRPPRC gene in the Leigh syndrome of the French-Canadian type (33).

In our group of children with isolated COX deficiency, more than $45 \%$ of patients presented with Leigh syndrome and had mutations in SURF1 gene. The deletion 845-846delCT in SURF1 gene was prevalent. On the contrary, the deletion 312-321del10insAT in SURF1 gene is prevalent in the nonSlavonic population of children with Leigh syndrome and COX deficiency (23). In children with encephalopathy and cardiomyopathy with mutations in $\mathrm{SCO} 2$ gene, the mutation $1541 \mathrm{G}>\mathrm{A}$ was found in all cases, making this mutation also prevalent, at least in our Slavonic population. We did not find any mutation in the COX10 gene analyzed in small subgroup of patients with renal disease, and no mutations in the SCOI gene were found in another small subgroup of patients with liver disease.

The most common genetic abnormalities in children with combined COX deficiency are usually linked to the mutations in mtDNA. In our patients with combined COX deficiency, the most harbored the large-scale mtDNA deletions and mtDNA point mutation $3243 \mathrm{~A}>\mathrm{G}$. Altogether, mtDNA mutations or deletions were found in $24 \%$ of our patients with combined COX deficiency. These numbers are much lower than in any group of adult patients with mitochondrial disorders, in whom a higher percentage of mtDNA mutations may be found (34). The increasing frequency of mtDNA mutations with respect to age may be explained by delayed onset of disease in the patients with mtDNA mutations in comparison with children with isolated COX deficiency caused by mutations in genes encoding the COX assembly factors. COX deficiency combined with other respiratory chain complex deficiencies may also arise from mutations in nuclear genes involved in mtDNA replication, translation, and transcription. Mutations in nuclear DNA were found for example in the POLG1 gene for mtDNA polymerase gamma (35) in children with Alpers syndrome and mtDNA depletion, in the gene for deoxyguanosine kinase (36) associated with hepatocerebral form of mtDNA depletion, and in the thymidine kinase- 2 gene (37) associated with myopathy. 


\section{CONCLUSIONS}

The results of our study suggest that COX deficiency in childhood is not rare in our Slavonic population. Understanding of the molecular basis of COX deficiencies in childhood is of key importance not only for noninvasive diagnostics, but for the genetic counseling and prenatal diagnostics in affected families.

\section{REFERENCES}

1. Hatefi Y 1985 The mitochondrial electron transport and oxidative phosphorylation system. Annu Rev Biochem 54:1015-1069

2. Taanman JW 1997 Human cytochrome c oxidase: structure, function, and deficiency. J Bioenerg Biomembr 29:151-163

3. Kadenbach B, Huttemann M, Arnold S, Lee I, Bender E 2000 Mitochondrial energy metabolism is regulated via nuclear-coded subunits of cytochrome c oxidase. Free Radic Biol Med 29:211-221

4. Huttemann M, Arnold S, Lee I, Muhlenbein N, Linder D, Lottspeich F, Kadenbach B 2000 Turkey cytochrome c oxidase contains subunit VIa of the liver type associated with low efficiency of energy transduction. Eur J Biochem 267:20982104

5. Schagger H, Noack H, Halangk W, Brandt U, von Jagow G 1995 Cytochrome-c oxidase in developing rat heart. Enzymic properties and amino-terminal sequences suggest identity of the fetal heart and the adult liver isoform. Eur J Biochem 230:235-241

6. Robinson BH 2000 Human cytochrome oxidase deficiency. Pediatr Res 48:581-585

7. Xu F, Morin C, Mitchell G, Ackerley C, Robinson BH 2004 The role of the LRPPRC (leucine-rich pentatricopeptide repeat cassette) gene in cytochrome oxidase assembly: mutation causes lowered levels of COX (cytochrome c oxidase) I and COX III mRNA. Biochem J 382:331-336

8. Skladal D, Sudmeier C, Konstantopoulou V, Stockler-Ipsiroglu S, Plecko-Startinig B, Bernert G, Zeman J, Sperl W 2003 The clinical spectrum of mitochondrial disease in 75 pediatric patients. Clin Pediatr (Phila) 42:703-710

9. Sacconi S, Salviati L, Sue CM, Shanske S, Davidson MM, Bonilla E, Naini AB, De Vivo DC, DiMauro S 2003 Mutation screening in patients with isolated cytochrome c oxidase deficiency. Pediatr Res 53:224-230

10. Berhman RE, Kliegman RM, Jenson HB 2004 Nelson Textbook of Pediatrics. Saunders, Philadelphia, p 59

11. Holmgren D, Wahlander H, Eriksson BO, Oldfors A, Holme E, Tulinius M 2003 Cardiomyopathy in children with mitochondrial disease; clinical course and cardiological findings. Eur Heart J 24:280-288

12. Wenchich L, Zeman J, Hansikova H, Plavka R, Sperl W, Houstek J 2002 Mitochondrial energy metabolism in very premature neonates. Biol Neonate 81:229-235

13. Makinen MW, Lee CP 1968 Biochemical studies of skeletal muscle mitochondria. I. Microanalysis of cytochrome content, oxidative and phosphorylative activities of mammalian skeletal muscle mitochondria. Arch Biochem Biophys 126:75-82

14. Rustin P, Chretien D, Bourgeron T, Gerard B, Rotig A, Saudubray JM, Munnich A 1994 Biochemical and molecular investigations in respiratory chain deficiencies. Clin Chim Acta 228:35-51

15. Srere PA 1969 Citrate synthase:[EC 4.1.3.7. Citrate oxaloacetate-lyase (CoAacetylating)]. Methods Enzymol 13:3-11

16. Lowry OH, Rosebrough NJ, Farr AL, Randall RJ 1951 Protein measurement with the Folin phenol reagent. J Biol Chem 193:265-275

17. Pecina P, Gnaiger E, Zeman J, Pronicka E, Houstek J 2004 Decreased affinity for oxygen of cytochrome-c oxidase in Leigh syndrome caused by SURF1 mutations. Am J Physiol Cell Physiol 287:C1384-C1388

18. Antonicka H, Floryk D, Klement P, Stratilova L, Hermanska J, Houstkova H, Kalous M, Drahota Z, Zeman J, Houstek J 1999 Defective kinetics of cytochrome c oxidase and alteration of mitochondrial membrane potential in fibroblasts and cytoplasmic hybrid cells with the mutation for myoclonus epilepsy with ragged-red fibres ('MERRF') at position 8344 nt. Biochem J 342:537-544

19. Pronicka E, Piekutowska-Abramczuk DH, Popowska E, Pronicki M, Karczmarewicz E, Sykut-Cegielska Y, Taybert J 2001 Compulsory hyperventilation and hypocapnia of patients with Leigh syndrome associated with SURF1 gene mutations as a cause of low serum bicarbonates. J Inherit Metab Dis 24:707-714
20. Pronicki M, Sykut-Cegielska J, Mierzewska H, Tonska K, Karczmarewicz E, Iwanicka K, Bartnik E, Pronicka E 2002 Diversity of clinical symptoms in A3243G mitochondrial DNA mutation (MELAS syndrome mutation). Med Sci Monit 8: CR767-773

21. Jesina P, Tesarova M, Fornuskova D, Vojtiskova A, Pecina P, Kaplanova V, Hansikova H, Zeman J, Houstek J 2004 Diminished synthesis of subunit a (ATP6) and altered function of ATP synthase and cytochrome c oxidase due to the mtDNA 2 bp microdeletion of TA at positions 9205 and 9206. Biochem J 383:561571

22. Tesarova M, Mayr JA, Wenchich L, Hansikova H, Elleder M, Blahova K, Sperl W, Zeman J 2004 Mitochondrial DNA depletion in Alpers syndrome. Neuropediatrics 35:217-223

23. Sue CM, Karadimas C, Checcarelli N, Tanji K, Papadopoulou LC, Pallotti F, Guo FL, Shanske S, Hirano M, De Vivo DC, Van Coster R, Kaplan P, Bonilla E, DiMauro S 2000 Differential features of patients with mutations in two COX assembly genes, SURF-1 and SCO2. Ann Neurol 47:589-595

24. Schaefer AM, Taylor RW, Turnbull DM, Chinnery PF 2004 The epidemiology of mitochondrial disorders-past, present and future. Biochim Biophys Acta 1659:115120

25. von Kleist-Retzow JC, Cormier-Daire V, de Lonlay P, Parfait B, Chretien D, Rustin P, Feingold J, Rotig A, Munnich A 1998 A high rate (20\%-30\%) of parental consanguinity in cytochrome-oxidase deficiency. Am J Hum Genet 63:428-435

26. Scaglia F, Towbin JA, Craigen WJ, Belmont JW, Smith EO, Neish SR, Ware SM, Hunter JV, Fernbach SD, Vladutiu GD, Wong LJ, Vogel H 2004 Clinical spectrum, morbidity, and mortality in 113 pediatric patients with mitochondrial disease. Pediatrics 114:925-931

27. Caruso U, Adami A, Bertini E, Burlina AB, Carnevale F, Cerone R, Dionisi-Vici C, Giordano G, Leuzzi E, Parenti G, Savasta S, Uziel G, Zeviani M 1996 Respiratorychain and pyruvate metabolism defects: Italian collaborative survey on 72 patients. J Inherit Metab Dis 19:143-148

28. Tiranti V, Corona P, Greco M, Taanman JW, Carrara F, Lamantea E, Nijtmans L, Uziel G, Zeviani M 2000 A novel frameshift mutation of the mtDNA COIII gene leads to impaired assembly of cytochrome c oxidase in a patient affected by Leigh-like syndrome. Hum Mol Genet 9:2733-2742

29. Williams SL, Taanman JW, Hansikova H, Houst'kova H, Chowdhury S, Zeman J, Houstek J 2001 A novel mutation in SURF1 causes skipping of exon 8 in a patient with cytochrome c oxidase-deficient leigh syndrome and hypertrichosis. Mol Genet Metab 73: 340-343

30. Valnot I, Osmond S, Gigarel N, Mehaye B, Amiel J, Cormier-Daire V, Munnich A, Bonnefont JP, Rustin P, Rotig A 2000 Mutations of the SCO1 gene in mitochondrial cytochrome $\mathrm{c}$ oxidase deficiency with neonatal-onset hepatic failure and encephalopathy. Am J Hum Genet 67:1104-1109

31. Vesela K, Hansikova H, Tesarova M, Martasek P, Elleder M, Houstek J, Zeman J 2004 Clinical, biochemical and molecular analyses of six patients with isolated cytochrome c oxidase deficiency due to mutations in the SCO2 gene. Acta Paediatr 93:1312-1317

32. Antonicka H, Mattman A, Carlson CG, Glerum DM, Hoffbuhr KC, Leary SC, Kennaway NG, Shoubridge EA 2003 Mutations in COX15 produce a defect in the mitochondrial heme biosynthetic pathway, causing early-onset fatal hypertrophic cardiomyopathy. Am J Hum Genet 72:101-114

33. Mootha VK, Lepage P, Miller K, Bunkenborg J, Reich M, Hjerrild M, Delmonte T, Villeneuve A, Sladek R, Xu F, Mitchell GA, Morin C, Mann M, Hudson TJ, Robinson B, Rioux JD, Lander ES 2003 Identification of a gene causing human cytochrome c oxidase deficiency by integrative genomics. Proc Natl Acad Sci U S A 100:605-610

34. Olsen DB, Langkilde AR, Orngreen MC, Rostrup E, Schwartz M, Vissing J 2003 Muscle structural changes in mitochondrial myopathy relate to genotype. J Neurol 250:1328-1334

35. Naviaux RK, Nyhan WL, Barshop BA, Poulton J, Markusic D, Karpinski NC, Haas RH 1999 Mitochondrial DNA polymerase gamma deficiency and mtDNA depletion in a child with Alpers' syndrome. Ann Neurol 45:54-58

36. Salviati L, Sacconi S, Mancuso M, Otaegui D, Camano P, Marina A, Rabinowitz S, Shiffman R, Thompson K, Wilson CM, Feigenbaum A, Naini AB, Hirano M, Bonilla E, DiMauro S, Vu TH 2002 Mitochondrial DNA depletion and dGK gene mutations. Ann Neurol 52:311-317

37. Saada A, Shaag A, Mandel H, Nevo Y, Eriksson S, Elpeleg O 2001 Mutant mitochondrial thymidine kinase in mitochondrial DNA depletion myopathy. Nat Genet 29:342-344 